\title{
A Self-Configurable Edge Computing for Industrial IoT
}

\author{
S. Chandramohan, M. Senthilkumaran
}

\begin{abstract}
The proliferation of Industrial Internet of Things (IIoT) introduces the concept of a smarter production environment. The emerging technologies like software defined network (SDN), IIoT and cloud computing will bring great advancements in the modern industrial revolution called Industry 4.0. Therefore, with the rapid development of IIoT technology, the proposed work incorporates with Edge Computing (EC). The current manufacturing process and automation, computing and wireless network reaches out to headways in innovation from easy to the point where all things (devices) and machines can interface through an Internet of Everything (IoE). This paper extends the work carried out in traditional methods, by integrating the cloud layer, Automatic Guided Vehicles (AGV), Industrial Wireless networks (IWN) and Industrial robots through EC is conferred to make autonomous decision-making capabilities. EC is emerging as a significant element in the smart industry to bring legacy in the context of Industrial IoT (IIoT). Finally, our proposed framework demonstrates that the active RFID-enabled AGV and industrial robots are brought in to exploit for effective resource management under the EC-based IIoT architecture, subsequently, it improves the conveyor efficiency and overall energy consumption in the warehouse for material handling.
\end{abstract}

Keywords: IoE, Edge Computing, Industrial Robot, AGV, Active RFID and IIoT.

\section{INTRODUCTION}

With the advancements in the today's industrial revolution such as SDN, Artificial Intelligence, Network Function Virtualization (NFV) and IoT technology, there is a great trade for smart manufacturing industry. In recent years, due to the rapid development of Industrial Wireless Sensor Networks, the manufacturing industry has reciprocated much in the form of the "Smart Industry". Also, the conventional industrial automation mechanisms are performed through wired communication. Despite, the wirebased automation systems depend upon expensive cables to be installed and expensive regular maintenance. Therefore, they are not generally implemented in current manufacturing industries. Accordingly, there is an urge towards effective wireless autonomous systems that provides optimized and efficient resource management on manufacturing systems [1].

Revised Manuscript Received on December 30, 2019.

* Correspondence Author

S. Chandramohan, Department of Electronics and Communication Engineering, Sri Chandrasekharendra Viswa Mahavidyalaya, SCSVMV Deemed to be University, Kanchipuram, INDIA. Email: chandramohan @ kanchiuniv.ac.in.

M. Senthilkumaran, Department of Computer Science and Engineering, Sri Chandrasekharendra Viswa Mahavidyalaya, SCSVMV Deemed to be University, Kanchipuram, INDIA. Email: msenthilkumaran @ kanchi univ. ac.in.

(c) The Authors. Published by Blue Eyes Intelligence Engineering and Sciences Publication (BEIESP). This is an open access article under the CC BY-NC-ND license (http://creativecommons.org/licenses/by-nc-nd/4.0/)
The conventional manufacturing industries are collecting the sensor data from various sources and uploads them to an industrial cloud and then the data to be processed. Also the conventional industrial automation mechanisms are realized though wired communication. Whereas, the present production industries produce a large amount of data from different devices and machines. Therefore, Industrial IoT as an effective communication network topology towards smart industry 4.0. An IoT device can receive and exchange data themselves through present communication network infrastructure connected by many wireless sensor nodes [11]. In the event that each IoT sensor essentially needs to process the information it gathers and sends result when certain prerequisites are met.

At that point, we begin to see the advantages of edge processing. The web of things (IoT) is the most well-known use case for edge computing [2]. Therefore, EC is appropriate for Industrial IoT deployments where both processing and localization could be utilized effectively [3].

\section{RELATED WORKS}

In this section, we comment briefly about an extant attempt regarding Edge Computing for energy-efficient resource management in the context of Industrial IoT.

Recently, Cisco popularized the concept of Fog Computing to implement in IoT applications on billions of things and devices towards a smarter environment, to run directly at the edge of the industrial wireless networks [4]. Eui-Nam Huh, et.al. [5] proposed a model for smart gateway and IoT resource estimation using fog computing. Stretching out this idea to an increasingly broad use case in IoT domain. An execution utilizing CloudSim demonstrated the viability of this work. Thus, applying the SDN and NFV ideal models will be helpful for this situation.

In this work, an author presented an edge-based model of a hybrid Cloud framework, which is intended to convey lowlatency and deliver efficient bandwidth. The data center networks are connecting through internal edge network, this model empowers less computation and unpredictable storage at the edge hubs. It gives the fundamental advantages like a specific decrease in latency for clients a long way from the data center and the data transmission decrease among edge and center gave by permitting the preprocessing at the edge cloud. The scope of this proposed work concludes to carry forward the cloud network near to the edge data centers [6]. 


\section{INDUSTRIAL AUTOMATION}

Industrial robot is a re-programmable and multi-useful administrator, intended to move materials, items, items and devices through modified movements. It might shift in like manner with the errand, which additionally get significant data and move cleverly accordingly [26], [27].

\section{A. Automated Guided Vehicle}

In the conventional industrial machines like $\mathrm{AGV}$ and Industrial robots have been working according to some set of standards. . These machines have the ability to change in the process to the computerized instrument. The Automatic Guided Vehicle is utilized and can be considered as the reasonable choice for control the utilization of the diverse thing's maneuvering. In a circumstance like essential automation inside a shop floor environment, it needs guidance, which is very important in medium and large scale industries. To deal with these unpredictable circumstances, for example, an adjustment in format for various conditions the AGV needs to receive keenly.

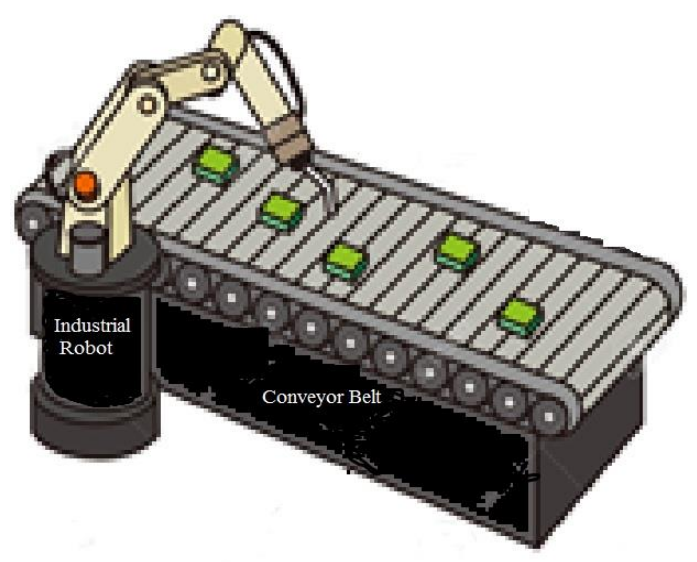

Fig 1. Autonomous Robots

Hence, this paper provides new direction for robots shown in figure 1 , and $\mathrm{AGV}$ to make continuously versatile in an intricate circumstance [12].

\section{B. Intelligent AGV and RBPA}

Automatic Guided Vehicles and Industrial robots are confronted with few limitations when they are deployed into a shop floor environment. Robotized Guided Vehicles have been used adequately for an item and material taking care of and for the most part for transportation inside and assembling industry. Regardless of this might be the perfect situation, actually a few undertakings request people and the shop floor are a blend of robotized that change of circumstances [7, 8]. Figure 2 shows the Robot-based Process Automation (RBPA) gives innovative answers for different sorts of organizations, working models that give computerization will upgrade the quality and effectiveness [9].

RBPA is the progressing methodology used to organize programming or an "Autonomous Robot" to translate existing applications for handling the product. It is changing the way in which we think in the business perspective [14, 15].

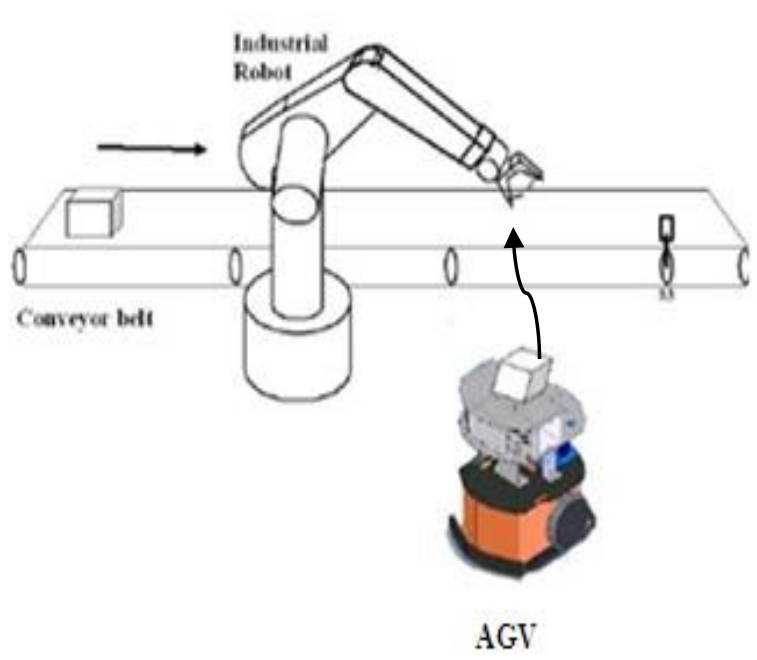

Fig 2. Integration of AGV and RBPA

Therefore, it gives extraordinary upgrades in exactness, process length and extended productivity by clearing repetitive task. In our proposed work, active RFID empowered AGV plays a vital role. RFID is utilized for direction, movement control and product identification. It transmits data through radio frequency waves of a particular frequency [16]. Thus, this paper centers on the procedure for planning an RFID enabled AGV method in SelfConfigurable IIoT framework [13].

\section{IIoT AND EDGE COMPUTING}

With the advancements in the current manufacturing industry such as smart manufacturing, augmented reality, SDN, Virtualization and a multitude of IoT applications, there is a great demand for an infrastructure with edge computing capabilities. In the EC [10], the processing of data occurs at the source rather than in a cloud. EC is merely computes similar to cloud computing [24]. To overcome few limitations in the conventional cloud computing technology, edge computing propels the computing at the edge devices to maneuver [23].

In a large scale industry, more sensors and devices are being interconnected through IoT gateway technology, and it will generate massive data and demand further processing [25]. In the conventional cloud computing method, to process these huge data via centralized severs will demand pressure on the data transmission cost, bandwidth allocation and other resources [20]. Furthermore, all IoT devices have limited power, it is compulsory to balance energy consumption by the devices that have higher power and computational capabilities.

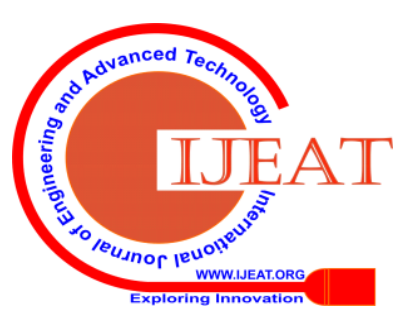




\section{EC FOR ENERGY RESOURCE MANAGEMENT}

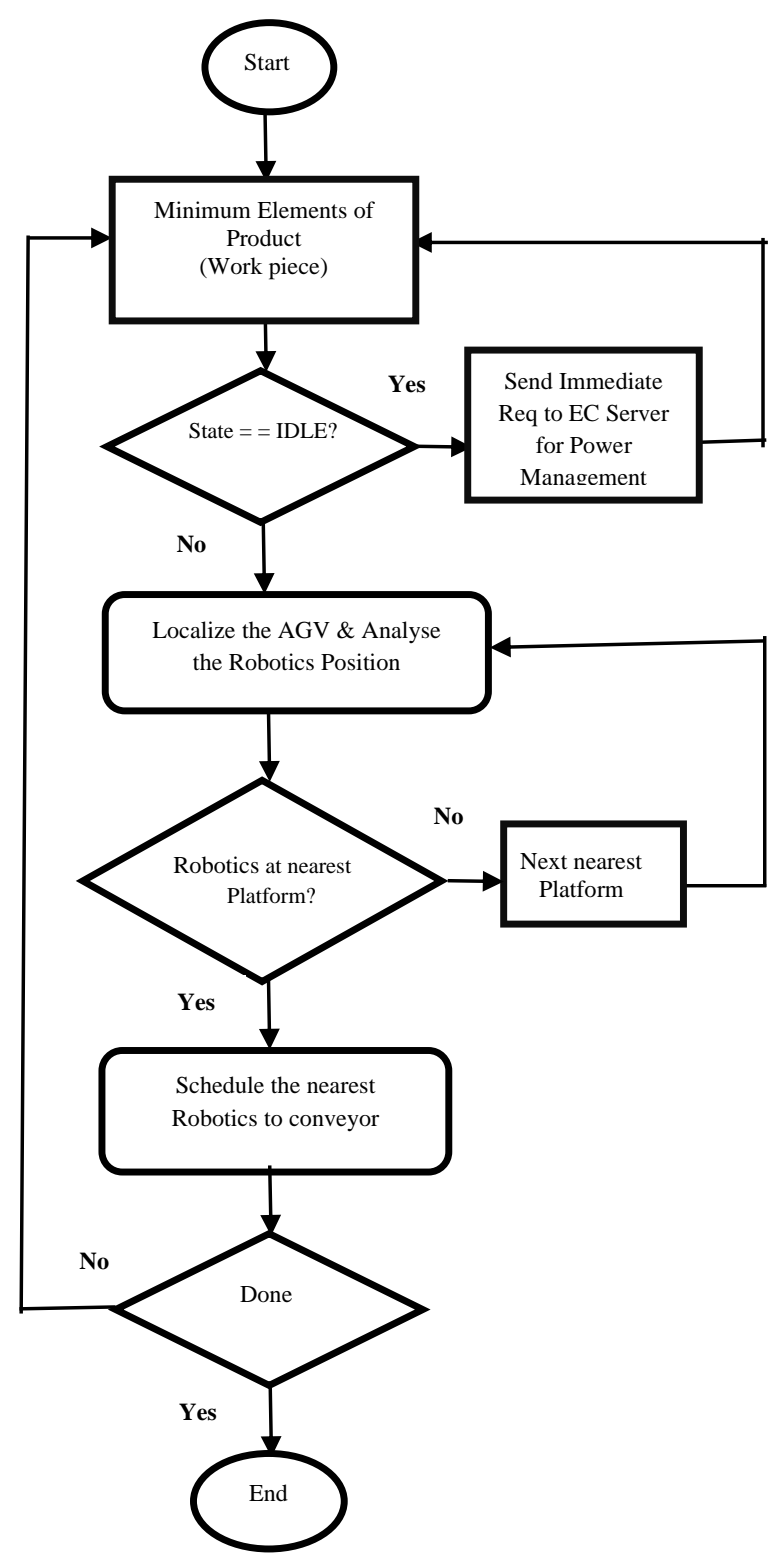

Fig. 3. Self-configurable Energy optimization mechanism.

EC for energy consumption involves various parameters, including edge computing information, shop floor details, AGV path, coordination mechanisms, sensor nodes, etc. Fig. 3 shows the edge computing procedure for effective management of power by analyzing the position of ActiveRFID enabled AGV.

In this scenario, all the robotics can access the EC server for scheduling the task. The task begins with minimum products that reflect the requirements for product handling and analyze the position of robotics at the nearest platform.

Now the instructions are forwarded to this robotics to complete the assigned task towards product delivery.

In the case of IDLE, that is no product in the queue, and then the status information sends the immediate request to EC server through the industrial wireless networks for the power management sector to take appropriate action.

Step by step, the program will find there is an AGV which is near to the platform. In case, there is no $\mathrm{AGV}$ is nearest to the platform, then the program will consider the next nearest platform, and reiterate. Once the AGV finds the nearest platform, the program reschedules the corresponding robots to deliver the product in a conveyor. Thus, our method is to finish the process in this cycle and continues further to the new cycle as per the instructions.

To overcome the aforementioned issues, we introduce our proposed method on edge computing for the Industrial IoT. EC carries the data computing that is outperformed at the "edge" of Industrial wireless networks. In addition, distributing computation nodes deployed at the edge can allow the offloading of traffic from the cloud for better response time [21], [22]. Thus, our proposed work reasonably decreases the transmission latency during data computing as well as power consumption in the SDN basedIIoT architecture with edge computing.

"Web of Things, Data and Service" is suggested by Germany Trade for the fourth industrial revolution called "Industry 4.0". In this generation a smarter industry provides a mechanical unrest from conventional assembling system to cyber physical system (CPS), [17]. Industrial IoT found with the benefits of enhanced creation, continuous observing and self-governing basic leadership towards smarter production [19].

Industrial IoT is a key component which unites present day distributed computing, Machine learning, and Artificial Intelligence to make canny, self-streamlining mechanical hardware and offices. In this way, the plan standards on brilliant item uses the most recent sensor innovation to upgrade different gear with remote observing abilities and its applications reaches out through information driven computerization rehearses in the modern segment of the economy. At long last, the IIoT thinks the need for assembling organizations as it enables them to give more an incentive to their clients. The Industrial Internet of Things (IIoT) relies upon information from sensors, remote systems and servers, regularly crosswise over collective, remote areas. Certain information preparing assignments are executed at source instead of in the cloud framework.

The Industrial Internet of Things (IIoT) depends on data from sensors, wireless networks and servers, often across collaborative, remote locations. Certain data processing tasks are executed at source rather than in the cloud infrastructure. 


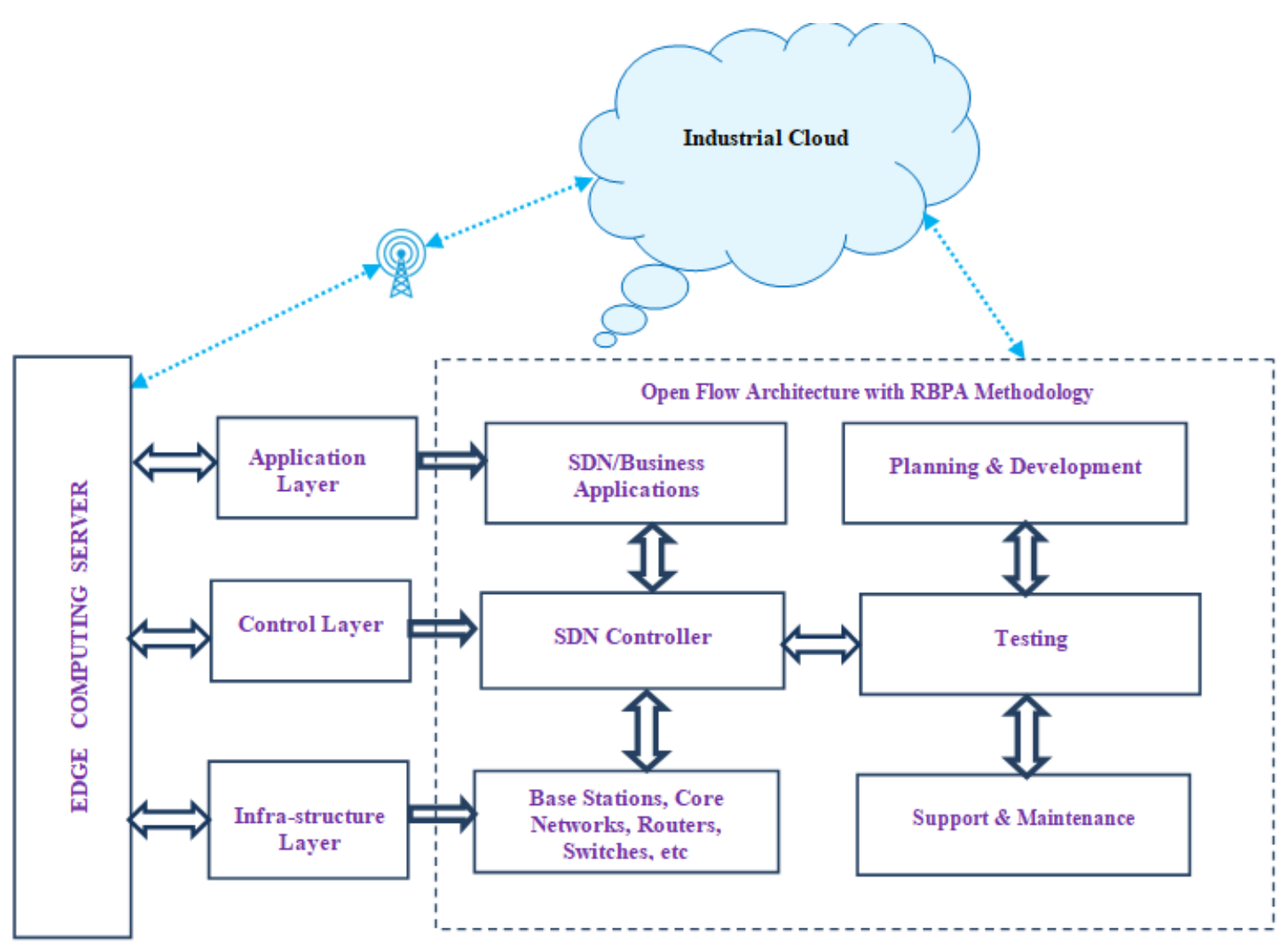

Fig 4. EC based Self Configurable IIoT Architecture

\section{RESULTS AND DISCUSSION}

We first review the correlations of related devices such as AGV, RBPA and Cyber-physical systems. A SelfConfigurable EC-driven architecture (fig.4) is proposed in the context of Industrial IoT to facilitate limited resource management. This paper extends the work carried out in [18], by integrating the cloud layer, Automatic Guided Vehicles (AGV), Industrial Wireless networks (IWN) and Industrial robots through EC is conferred to make autonomous decision-making capabilities, and this section explains the simulation setup, performance metrics, and emulation scenarios.

This work is developed by the simulation work and realized the algorithm in MATLAB environment. To evaluate the energy optimization and efficiency of the proposed work, we introduce the following aspects.

[1]. Overall Time Delay (OTD): The OTD represents the time required for product delivery from the warehouse to a conveyer belt.

[2]. Quality of Service (QoS): The QoS performance metric is expressed as effective utilization of resources.

[3]. Throughput: It is the rate of successful product delivery over a warehouse, in Industrial IoT. This performance measurement has the advantage for better evaluation of successful data rate receiving from warehouse to destination (conveyor).
Table: 1 Comparison of Throughput and Energy Efficiency

\begin{tabular}{|c|c|c|}
\hline Methods & $\begin{array}{c}\text { Utilization Rate } \\
\text { (Work Piece) }\end{array}$ & $\begin{array}{c}\text { Energy } \\
\text { Consumption(W) }\end{array}$ \\
\hline Traditional & 01 & 2 \\
\hline SD-IIoT [18] & 02 & 1 \\
\hline Proposed & 02 & 0.75 \\
\hline
\end{tabular}

Table 1 shows the performance comparison of various methods, among all our work reduce the energy effectively. Since complex tasks can be offloaded to the IWNs and cloud infrastructure, this could limit the energy constraints. Software-Defined-IIoT has been different manifestations, for example, Machine to Machine communication and industrial networks still facing with constraints. Whereas Industrial Robot is as yet having a few impediments. The proposed work improves the conveyer efficiency and overall energy consumption decreased reasonably, when compared with SD-IIoT [18]. In the material handling process at conveyer belt, our intelligent AGV loads parallel along with the fixed Industrial robots. Therefore our proposed work utilizes the resources effectively. Figure 4 shows the Energy consumption is decreased when compared with SD-IIoT architecture. 


\section{Energy Consumption}

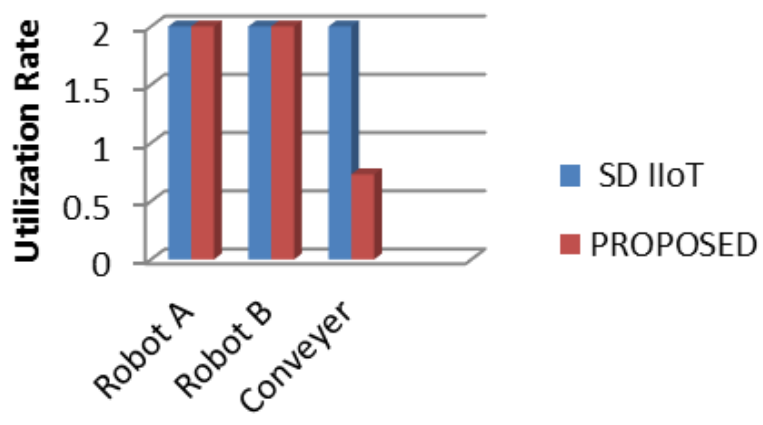

Fig. 5. Comparison of Energy Efficiency

\section{CONCLUSION}

With the advancements in the current manufacturing industry such as smart manufacturing, augmented reality, SDN, Virtualization and a multitude of IoT applications, there is a great demand for an infrastructure with edge computing capabilities. The industry 4.0 represents the basis of smart production company. With the scope to improve the effective resource management, A Self-Configurable EC-driven architecture is proposed. The obtained results have demonstrated that our proposed work improves the conveyor efficiency and overall energy consumption. Thus, the EC based IIoT architecture will facilitate in the context of Industry 4.0.

\section{REFERENCES}

1. Gungor V.C and P Hanck G, "wireless sensor networks, Design principles, chanllenges and technical approachs," vol. 56, no. 10, pp. 4258-4265-IEEE Transaction.Ind.Electron, Oct-2009.

2. Wan.J, Imran Mohammmad, Wang, D. Li, and C. Zhang, “ Using cloud smart manufacturing for personalized candy packing application, pp. 1-19, Sep. 2016, DOI: 10.1007/s11227-016-1879-4.

3. J. Liu et al., " The Scalable and fast response SD- vehicular network assisted by MEC”, vol. 55, no.7,pp. 94-100, Communication Magazine IEEE - July 2017

4. Addepalli sant, Bonomi fern, Milito, J. Zhu, "Fog computing and its role in the internet of things", MCC workshop on Mobile cloud computing, August 2012.

5. H. Eui-Nam, and Aazam and Mo, "Fog Computing Micro Datacenter Based Dynamic Resource Estimation and Pricing Model for IoT," Advanced Information Networking and Applications (AINA), 2015 IEEE 29th International Conference on, pp. 687-694

6. Hari, Chang H, S. Mukherjee and T.V. Lakshman, "Bringing the cloud to the edge," Computer CommunicationsWorkshops (infocom wkshps), IEEE Conference on, pp. 346-351- 2014.

7. Zhong, Klotz, S. T. Newman, "Review: A Intelligent Manufacturing with respect to Industry 4.0" Elsevier Engineering, vol. 3, pp. 616-630, 2017.

8. Wang.h, Xu, R.Y. Zhong, S.P. Lu,"RFID using positioning system in automated guided vehicle for smart Industries," Journal of Manufacturing Systems, vol. 44, pp. 179-190 - 2017.

9. D.Luo, Ying, Pasquale Pace, Wenfeng $\mathrm{Li}$ and Giancarlo Fortino, "Workshop Networks Integration Using Mobile Intelligence in Smart Factories", 0163-6804/18/\$25.00 @ 2018 IEEE, IEEE Communications Magazine - 2018

10. A kayssi, Salman, O.Imad Elhaj, Ali Chehab, "Edge Computing Enabling the Internet ofThings",DOI:10.1109/AICCSA.2015.7507265IEEE International Conference of Computer Systems and Applications $-2015$

11. Wan.J, D. Zhang Li.D ,Zhang. C Wang, J, “A self organized multiagent system assisted with big data based feedback and coordination: Towards the smart factory" Elsevier, - Computer Networks., vol. 101, pp. 158-168, Jun 2016.

12. Mehami, R.Y Zhong and Mauludin.N, "AGV for manufacturing towards Smart Industry 4.0" 2351-9789, Elsevier- North American Manufacturing Research Conference- 2018.

13. Xu,Zhong.RY, and Wang.H , " IoT-Enabled Real time Machine Status Monitoring Approach for Cloud Manufacturing," Procedia CIRP, vol. 63, pp. 709-714, 2017.

14. Shenglong .T, Chengliang.L, J. Lloret,,Jiafu Wan and Qingsong Hua,,“ Cloud Robotics for Material Handling in Cognitive Industrial Internet of Things", Internet Of Things Journal, VOL.5, NO. 4, IEEE - Aug, 2018

15. Shaoping Lu, Zhong, and Chen $\mathrm{Xu}$,“Active RFID tag using locating Approach With Multipath Effect Elimination in AGV" VOL. 13, NO. 3, july 2016- IEEE Transactions on Automation.

16. S.Lee, Harashima.F, K. C. Lee and Lee.H, " The integration of vehicles (mobile) for automated material handling using IEEE Networks and Profibus," vol. 49, no. 3,pp. 693-701, Jun. 2002.

17. N. Jazdi, "Cyber physical systems in the context of industry 4.0," in Proc. IEEE Int. Conf. Autom. Quality Testing Robot., 2014, pp. 1-4.

18. Li Di, Sheng T. long, Jiafu Wan, Member IEEE, Zhaogang S,Shiyong Wang and V.Athanasios, "Software Defined Industrial Internet of Things in the Context of Industry 4.0", IEEE Sensor Journal, vol. 16, no. 20, October 15, 2016

19. Ahmad , Jiafu, Chen.B, M.Imran, Di Li, and Chengliang Liu, “ Towards Dynamic Resource Management for IoT Based Manufacturing”, IEEE Communication 0163-6804/18/2018, February 2018.

20. J. Wu and W. Zhao, "Design and realization of WInternet: From net of things to Internet of Things," ACM Trans. Cyber-Phys. Syst., vol. 1, no. 1,pp.2:1_2:12,Nov.2016. [Online]. http://doi.acm.org/10.1145/2872332

21. M. Jutila, "An adaptive edge router enabling Internet of Things," IEEE Internet Things J., vol. 3, no. 6, pp. 1061_1069, Dec. 2016.

22. D. Georgakopoulos, P. P. Jayaraman, M. Fazia, M. Villari, and R. Ranjan, Internet of Things and edge cloud computing roadmap for manufacturing, IEEE Cloud Comput., vol. 3, no. 4, pp. 66_73, Jul./Aug. 2016.

23. J. Liu et al., "A scalable and quick-response software defined vehicular network assisted by mobile edge computing," IEEE Commun. Mag.,vol. 55, no. 7, pp. 94-100, Jul. 2017.

24. http://www.networkcomputing.com/networking/how-edge-computingcompares-cloudcomputing

25. K.Byungseok, Kim Daecheon, Hyunsung Choo, "Internet of Everything: A Large-Scale Autonomic IoT Gateway", IEEE transactions on multi-scale computing systems, vol. 3, no. 3, julyseptember 2017.

26. K.Sung, I. Lee, H. Song, Jung.J, "AGV System for Vision guidance using-ANFS," International Conference on Intelligent Robotics and Applications pp. 377-38- 2012

27. Nielsen. "Implementation Methodology of Mobile Robot in Adaptive Manufacturing Environments," vol. 28, no. 5, 2017, pp. 1171-88.

\section{AUTHORS PROFILE}

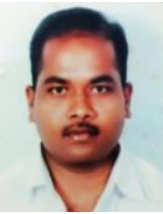

S. Chandramohan, obtained his BE degree in Electronics and Communication Engineering from ANNA University in the year 2002. He completed his M.Tech degree in Space Technology and Applications from Indian Institute of Science, Bangalore and Pursuing $\mathrm{PhD}$ from SCSVMV (Deemed to be University), Kanchipuram. He has nearly ' 15 ' years of Experience in the field of Engineering Education and Administration. He has published 07 papers in Journals and Conferences both at the level of National and International. He is a member of various professional bodies such as IEEE and IAENG. Currently he is working as Assistant Professor in the Department of ECE, SCSVMV (Deemed University), Kanchipuram.

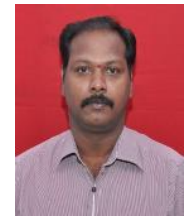

M. Senthil Kumaran, is working as an Associate Professor in the department of Computer Science and Engineering of SCSVMV, Enathur, Kanchipuram. He has published 25 papers in International Journals, presented more than 20 papers in international and National Conferences. He is a reviewer of International Journal of Communication Systems, Journal of Computer Science and International Journal of Computer Science Issues. He is a member in various societies such as Computer Society of India, IAENG, ISTE, IBM my Developer works Community and senior member of IACST. 